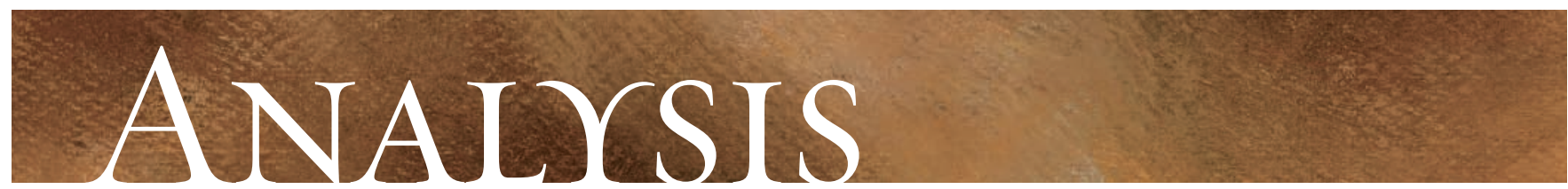

\section{Dientamoeba fragilis:}

\section{an emerging role in}

\section{intestinal disease}

$\mathrm{F}$ irst described in I9I8 by Jepps and Dobell, Dientamoeba fragilis is a binucleated, unflagellated protozoan related to the trichomonads, ${ }^{1}$ readily identified in stool specimens by means of routine iron-hematoxylin stains. First observed in 7 patients, of whom 6 had diarrhea or dysentery, the parasite was dubiously classified as a nonpathogen based on its source of nutrition: its voracious appetite is for the commensal bacteria of the gut rather than the tissues of its host. ${ }^{1}$ For decades, the organism was thrown into the grab-bag of human commensal protozoa with the likes of Entamoeba coli and Endolimax nana. Questions about the nonpathogenicity of this protozoan have emerged, however, and there can now be little doubt of its role in gastrointestinal disease. ${ }^{1-3}$

Only recently has the evidence supporting $D$. fragilis as a cause of diarrhea, abdominal pain, cramping and a plethora of vague abdominal com-

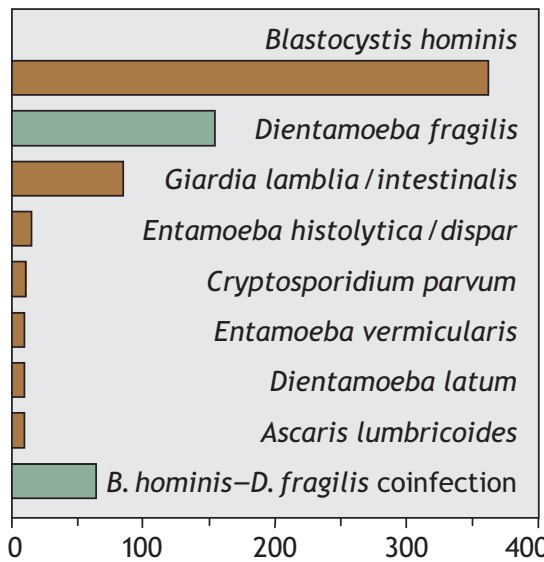

No. of specimens positive for parasites

Fig. 1: Nonrepeat occurrence of the 8 most common intestinal parasites diagnosed via stool examination at Cadham Provincial Laboratory, Winnipeg, Man., over a 1-year period. plaints come to light. The organism has been isolated from patients with clinical disease in countries around the world, including an Australian study reporting that all of 60 patients with confirmed $D$. fragilis infection were symptomatic. ${ }^{3}$ Moreover, treatment with drugs known to have parasiticidal activity in vitro has led to prompt and dramatic clinical improvement in the majority of reported cases. ${ }^{1,2}$ In some cases, patients with prior misdiagnoses of irritable bowel syndrome (IBS) or chronic diarrhea have been found to be infected with the parasite and cured with antiparasitic agents. ${ }^{1}$ Such observations indicate a need not only for appropriate investigation but also treatment of patients who have symptoms of irritable bowel syndrome before a reputedly incurable condition is diagnosed. As a result of these and other observations, many countries have finally recognized $D$. fragilis as a true gastrointestinal pathogen. ${ }^{1}$

The prevalence of $D$. fragilis would surprise most clinicians. A review of stool-examination reports at the Cadham Provincial Laboratory in Winnipeg, which handles $80 \%-90 \%$ of stool examinations for parasites done in Manitoba, revealed that the incidence of this parasite was second only to Blastocystis hominis and far in excess of more commonly incriminated parasites such as Giardia lamblia/intestinalis, Entamoeba histolytica/dispar and Cryptosporidium parvum. Coinfection with $B$. hominis and $D$. fragilis was also common (Fig. I).

To examine the epidemiology of $D$. fragilis infection in Manitoba, we reviewed the examination results for all fecal specimens submitted over a I2month period (Feb. 2005 through Jan. 2006) to the Cadham Provincial Laboratory for ova and parasites: a total of II Ioo specimens from $6_{3} 6_{3}$ patients (mean 1.74 specimens per patient). The parasite was found predominantly in specimens from young adults: $50 \%$ of people found to have dientamoebiasis in Manitoba were younger than 24 (mean 28.7) years. This contrasts with a median age of $4 \mathrm{I}$ years for all patients submitting samples (mean $40.8 \mathrm{yr}$ ), which suggests that sample bias does not account for the observation of differences in the incidence of this parasite in younger people.

Specimens from patients II-I5 years of age had relatively high rates of positive reports (boys 10.3\%, girls 9.6\%). Those from young men aged $16-20$ years had the highest rate: 1 II. $5 \%$ (95\% confidence interval [CI] 4\%-I $\% \%$ ). The positivity rate of specimens from young women aged $16-20$ years, on the other hand, was much lower (I.I\%, 95\% CI $0.3 \%-\mathrm{I} .9 \% ; p<0.0 \mathrm{I})$, in contrast with reports from other countries ${ }^{1,2}$ where a female predilection has been suggested. Furthermore, $45.9 \%$ of positive results applied to specimens from female patients, who furnished $58.3 \%$ of the specimens submitted $(p=0.03)$. Adults over 20 years of age had the lowest incidence rates $(0.6-2.0 \%)$; the rates in children under to were intermediate (I. $3 \%-5.7 \%$ ). Incidence rates showed no seasonal variation over a 5 -year period (data not shown), which contrasts sharply with the summer-fall seasonality of pathogens traditionally associated with contaminated water (e.g., G. lamblia/intestinalis, C. parvum).

The mode of transmission of $D$. fragilis remains a mystery, perhaps because its acknowledgement as a pathogen is so recent. The organism has never been found to have a cyst stage, deemed necessary for efficient feco-oral transmission. ${ }^{1-3}$ Moreover, direct transmission via trophozoite forms was deemed unlikely when, in keeping with a longstanding practice among early parasitologists of self-experimentation, Dobell swallowed a culture containing millions of trophozoites. ${ }^{1}$ Not only did he remain asymptomatic, but for 10 years he examined his own stool specimens and never observed the parasite.

Because of its morphological, biological and genetic similarities to protozoan parasites of nonhuman animals that are known to use the eggs of parasitic worms as vectors, it was speculated 
Table 1: Treatments that have exterminated Dientamoeba fragilis*

\begin{tabular}{lcc}
\hline Agent & Dose, mg $\dagger$ & Duration, d \\
\hline $\begin{array}{l}\text { lodoquinol } \\
\text { Doxycycline, }\end{array}$ & 650 & 20 \\
twice a day & 100 & 10 \\
\hline $\begin{array}{l}\text { Metronidazole } \\
\text { Paromomycin, } \\
\text { mg/kg body wt }\end{array}$ & 500 & 10 \\
\hline $\begin{array}{l}\text { Secnidazole, } \\
\text { single dose }\end{array}$ & 2000 & 7 \\
\hline
\end{tabular}

Note: $w t=$ weight.

*Adapted from references 1, 2 and 5-9.

†Administered by mouth: 3 times per day, unless otherwise indicated.

that $D$. fragilis may also be transmitted that way. ${ }^{1}$ Early studies pointed toward the pinworm, Enterobius vermicularis, as a potential vector: coinfection with $D$. fragilis and E. vermicularis initially appeared to be far more common than expected; amoeboid bodies resembling $D$. fragilis have been described in the eggs of $E$. vermicularis; and the parasitologist Ockert (again, in the tradition of self-experimentation) successfully infected himself and 2 other adult volunteers by ingesting $E$. vermicularis eggs taken from a child coinfected with $D$. fragilis. ${ }^{1}$

Despite these observations, many have questioned a role for pinworms in transmission, frequently citing the paucity of well-controlled epidemiological experiments. ${ }^{1}$ In one study, Stark and colleagues $^{3}$ performed appropriate pinworm testing in conjunction with microscopic stool examination and found no association between infection with Enterobius and Dientamoeba. The authors acknowledged, however, that spontaneous remission of pinworm infection could have occurred while $\mathrm{Di}$ entamoeba infection persisted. Similarly, our study did not identify any co- infections with Enterobius and Dientamoeba, but appropriate testing (sticky tape or paddle) for Enterobius eggs was not performed consistently. Lastly, PCR of nucleic acid extract of Enterobius eggs did not identify Dientameoba DNA in coinfected individuals. ${ }^{4}$ As it stands, although the mode of transmission remains unknown, it seems fair to query the role of Enterobius in the transmission of Dientamoeba.

Several drugs are thought to have paraciticidal activity against $D$. fragilis. Unfortunately, in vitro testing is inaccurate and cannot reliably predict treatment outcomes because Dientamoeba requires xenic media (media containing bacteria for the parasite to feed on). Furthermore, no large-scale randomized control trials have been done. Many agents have, however, led to the eradication of $D$. fragilis from stools and resolution of symptoms as documented in case reports (Table I). ${ }^{1,2,5-9}$ The most commonly employed and best studied treatments currently are iodoquinol and doxycycline. ${ }^{1,8}$ This regimen was studied in a small case series of 2I people with IBS-like symptoms and concomitant infection with $D$. fragilis who were treated with iodoquinol and doxycycline: the symptoms resolved in $\mathrm{I} 4$ of the patients $(67 \%)$, in whom the organism was eradicated. In a more recent study of secnidazole treatment, ${ }^{9}$ a single dose eradicated the parasite in 34 of 35 patients $(97 \%$, 95\% CI 92\%-I00\%) and resolved clinical symptoms in 27 cases $(77 \%, 95 \%$ CI 63\%-9I\%).

Intestinal parasites, particularly those found in temperate climates, are often forgotten as causes of disease. Increasing evidence of pathogenicity in common parasites previously deemed as nonpathogenic, including $D$. fragilis, should prompt clinicians to consider eradicating $D$. fragilis when it is found in all patients with gastrointestinal complaints. Furthermore, people who have symptoms of IBS should be evaluated for the presence of this and other parasites. Where required, treatment should be provided before a diagnosis of IBS is given.

\section{Philippe R. Lagacé-Wiens}

Department of Medical Microbiology and Infectious Diseases

University of Manitoba

Paul G. VanCaeseele

Department of Medical Microbiology and Infectious Diseases

University of Manitoba

Cadham Provincial Laboratory

Cliff Koschik

Cadham Provincial Laboratory

Winnipeg, Man.

This article has been peer reviewed.

Competing interests: None declared.

\section{REFERENCES}

I. Johnson EH, Windsor JJ, Clark CG. Emerging from obscurity: biological, clinical, and diagnostic aspects of Dientamoeba fragilis. Clin Microbiol Rev 2004;17:553-70.

2. Norberg A, Nord CE, Evengard B. Dientamoeba fragilis - a protozoal infection which may cause severe bowel distress. Clin Microbiol Infect 2003; $9: 65-8$.

3. Stark D, Beebe N, Marriott D, et al. Prospective study of the prevalence, genotyping, and clinical relevance of Dientamoeba fragilis infections in an Australian population. J Clin Microbiol 2005;43: 2718-23.

4. Menghi CI, Makiya R, Gatta CL, et al. Dientamoeba fragilis: molecular biology techniques for the elucidation of its mode of transmission. Parasitol Latinoam 2005;60:25-3I.

5. Dardick KR. Tetracycline treatment of Dientamoeba fragilis. Conn Med I983;47:69-70.

6. Spencer MJ, Garcia LS, Chapin MR. Dientamoeba fragilis: an intestinal pathogen in children? Am J Dis Child 1979;133:390-3.

7. Cuffari C, Oligny L, Seidman EG. Dientamoeba fragilis masquerading as allergic colitis. J Pediatr Gastroenterol Nutr 1998;26:16-20.

8. Borody T, Warren E, Wettstein A, et al. Eradication of Dientamoeba fragilis can resolve IBS-like symptoms. J Gastroenterol Hepatol 2002;17(Suppl):Aro3.

9. Girginkardesler N, Coskun S, Cuneyt Balcioglu I, et al. Dientamoeba fragilis, a neglected cause of diarrhea, successfully treated with secnidazole. Clin Microbiol Infect 2003;9:110-3.

\section{IMPACT}

$C M A J$ is a leading international general medical journal as ranked by Thomson ISI. 2005 impact factor, 7.4. 